\title{
The role of the electronic current component in the formation of a quasi-molecular state leading to the synthesis of elements
}

\author{
M.P. Kashchenko ${ }^{\dagger, 1,2}$, N. M. Kashchenko ${ }^{1}$ \\ †mpk46@mail.ru \\ ${ }^{1}$ Ural Federal University n. a. the first President of Russia B. N. Yeltsin, 19 Mira St., Yekaterinburg, 620002, Russia \\ ${ }^{2}$ Ural State Forest Engineering University, 37 Sibirskii Tract, Yekaterinburg, 620100, Russia
}

\begin{abstract}
It was previously shown that in the process of plasma water electrolysis, a synthesis of chemical elements is observed indicating the existence of low-energy nuclear fusion reactions. In the traditional consideration, for guaranteed fusion of nuclei, their approach to a distance of the order of the nucleus size $R_{n} \sim 10^{-15} \mathrm{~m}$ is required. An additional possibility is to use electromagnetic interaction to achieve an intermediate quasimolecular state with a critical internuclear distance of $R_{c} \sim 10^{-13} \mathrm{~m}$, which is smaller than Bohr radius $R_{\mathrm{B}} \approx 5 \cdot 10^{-11} \mathrm{~m}$, but larger $R_{n}$. When $R_{c}$ is reached, the process of attraction of the nuclei becomes possible due to the exchange of virtual electron-positron pairs, the efficiency of which increases with the approach of the nuclei. Since in the framework of the hadronic mechanics of Santilli, the $\pi^{0}$-meson is interpreted as a result of the contact interaction of an electron and a positron, the stage of approach of the nuclei from $R_{c}$ to $R_{n}$ due to the exchange of quasipositroniums can be considered as an extension of the action of the Yukawa mechanism on scales up to $R_{c}$. Thus, the approach of nuclei to $R_{c}$ plays a key role in the implementation of nuclear fusion. A similar approach is possible if a high electron density arises between the nuclei in the process of inelastic collision of ions (atoms). In the model of an intermediate quasimolecular state, an increase in the internuclear density of electrons is considered to be a consequence of the formation of pair Bose-type electronic states arising from the contact interaction (attraction) of electrons at the femt scale as shown in hadron mechanics. Therefore, the electronic component of the current during the electrolysis of solutions should contribute to the synthesis of elements by initiating the formation of Bose electron pairs in tunable shells of ions (atoms). This conclusion is confirmed by estimates of the transparency coefficient for electron tunneling through the Coulomb barrier. The transparency coefficient for the tunneling of hydrogen nuclei in muon catalysis is estimated. The possibility of the occurrence of simple nuclear reactions during the interaction of the initial nuclei with quasineutrons is noted.
\end{abstract}

Keywords: synthesis of elements, intermediate quasimolecular state, quasipositronium, tunneling effect, Bose electron pairs.

УДК: 539.17

\section{Роль электронной составляющей тока в образовании квазимолекулярного состояния, ведущего к синтезу элементов}

\footnotetext{
Кащенко М. П. ${ }^{\dagger, 1,2}$, Кащенко Н. М. ${ }^{1}$

${ }^{1}$ Уральский федеральный университет им. первого президента России Б. Н. Ельцина, ул. Мира, 19, Екатеринбург, 620002, Россия

${ }^{2}$ Уральский государственный лесотехнический университет, Сибирский тракт, 37, Екатеринбург, 620100, Россия

Ранее было показано, что в процессе плазменного электролиза воды наблюдается синтез химических элементов, указывающий на существование низкоэнергетических ядерных реакций синтеза. При традиционном рассмотрении для гарантированного слияния ядер требуется их сближение на расстояние порядка размера ядра $R_{n} \sim 10^{-15}$ м. Дополнительная возможность состоит в использовании электромагнитного взаимодействия для достижения про-
} 
межуточного квазимолекулярного состояния с критическим межъядерным расстоянием $R_{c} \sim 10^{-13}$ м существенно меньшим боровского радиуса $R_{\mathrm{B}} \approx 5 \cdot 10^{-11} \mathrm{M}$, но большим $R_{n}$. При достижении $R_{c}$ становится возможным процесс притяжения ядер за счет обмена виртуальными электрон-позитронными парами, эффективность которого растет по мере сближения ядер. Поскольку в рамках адронной механики Сантилли $\pi^{0}$-мезон интерпретируется как результат контактного взаимодействия электрона и позитрона, этап сближения ядер от $R_{c}$ до $R_{n}$ за счет обмена квазипозитрониями можно рассматривать как расширение действия механизма Юкавы на масштабы вплоть до $R_{c}$. Таким образом, сближение ядер до $R_{c}$ играет ключевую роль для реализации ядерного синтеза. Подобное сближение оказывается возможным, если между ядрами в процессе неупругого столкновения ионов (атомов) возникает высокая электронная плотность. В модели промежуточного квазимолекулярного состояния возрастание межъядерной плотности электронов считается следствием образования парных электронных состояний бозевского типа, возникающих при контактном взаимодействии (притяжении) электронов на фемтомасштабе, как показано в адронной механике. Следовательно, электронная составляющая тока при электролизе растворов должна способствовать синтезу элементов за счет инициирования формирования бозевских электронных пар в перестраиваемых оболочках ионов (атомов). Данный вывод подтверждается оценками коэффициента прозрачности для туннелирования электронов через кулоновский барьер. Приводится оценка коэффициента прозрачности для туннелирования ядер водорода при мюонном катализе. Отмечается возможность протекания простейших ядерных реакций при взаимодействии исходных ядер с квазинейтронами.

Ключевые слова: синтез элементов, промежуточное квазимолекулярное состояние, квазипозитроний, туннельный эффект, бозевские пары электронов.

\section{1. Введение}

Образование гелия при реакции мюонного катализа $[1,2]$ показало возможность протекания низкотемпературного синтеза в присутствии отрицательно заряженного мюона. Это позволило предположить [3], что наблюдаемые варианты низкоэнергетических ядерных реакций $[4,5]$ и особенно синтеза более массивных элементов [6,7] связаны с возникновением промежуточных квазимолекулярных состояний (ПКС), характеризуемых высокой электронной плотностью в пространстве между исходными ядрами. В [8] была предложена модель ПКС (см. Рис. 1), в которой высокая электронная плотность в межъядерной области рассматривается как следствие образования парных электронных состояний бозевского типа, возникающих при контактном взаимодействии (притяжении) электронов [9] на адронных масштабах $R_{n} \sim 10^{-15}$ м.

Существенно, что в модели ПКС допускается сближение ядер до критического расстояния $R_{c} \sim 10^{-13}$ м почти на три порядка меньшего характерного боровского радиуса $R_{\mathrm{B}} \approx 5 \cdot 10^{-11}$ м, но еще на два порядка большего $R_{n}$. Как отмечено в [8], концепция виртуальных частиц,

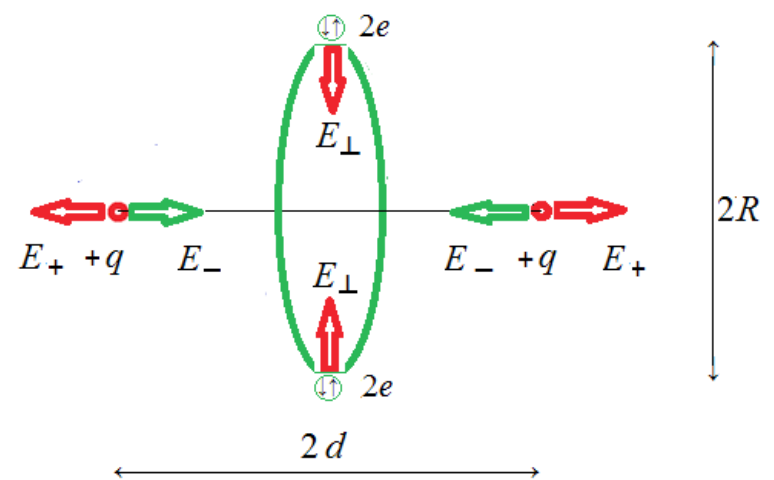

Puc. 1. (Color online) Схема простейшей модели ПКС [8].

Fig. 1. (Color online) The scheme of the simplest IQS model [8]. базирующаяся на соотношениях неопределенности Гейзенберга для энергии и времени и широко используемая в квантовой механике (см. например, [10]), показывает, что при достижении $R_{c}$ становится возможным процесс притяжения ядер за счет обмена виртуальными электронпозитронными парами, эффективность которого растет по мере сближения ядер. Поскольку в рамках адронной механики Сантилли $\pi^{0}$-мезон интерпретируется как результат контактного взаимодействия электрона и позитрона [9], этап сближения ядер от $R_{c}$ до $R_{n}$ за счет обмена квазипозитрониями можно рассматривать как расширение применимости механизма Юкавы [11] на масштабы вплоть до $R_{c}$. Таким образом, сближение ядер до $R_{c}$ играет ключевую роль для реализации ядерного синтеза.

В предыдущих работах $[3,8]$ не обсуждалась роль электронной составляющей электрического тока. Между тем, в оригинальной методике (глава 4 в [6], далее под [6] имеется в виду эта глава) синтеза элементов при плазменном электролизе воды электрические токи могли достигать 120 А и наверняка содержали значительную электронную составляющую. В свете модели ПКС роль этой составляющей представляется весьма существенной для формирования в атомах (ионах) состояний с высокой электронной плотностью за счет образования электронных пар, подчиняющихся бозевской статистике. Подобные пары должны становиться катализаторами быстрой перестройки электронного спектра атомов (ионов), необходимой для формирования ПКС при столкновении атомов (ионов). Однако возникновение пары связанных электронов требует преодоления потенциального барьера, связанного с их кулоновским отталкиванием.

Цель данной работы - провести оценки коэффициента прозрачности кулоновского барьера при сближении двух электронов до расстояний $\sim R_{n}$ и обсудить полученную информацию для уточнения представлений о механизме формирования ПКС. 


\section{2. Оценка коэффициента прозрачности при туннелировании свободного электрона, необходимом для образования контактно взаимодействующей пары электронов}

В работе [6], как и в наших работах [12-14], типичные значения разности потенциалов составляли 500 В. Поэтому при оценках естественно ориентироваться на кинетические энергии электронов $E$ до 0.5 кэВ.

На Рис. 2 приведена используемая далее простая модель прямоугольной потенциальной ямы (притяжение для области ямы) с чисто кулоновским отталкиванием $U_{\mathrm{C}}(r)$ вне ямы (начало отсчета потенциала выбирается в бесконечно удаленной точке):

$$
U_{\mathrm{C}}(r)=e^{2} \frac{1}{4 \pi \varepsilon_{0} r},
$$

где $\varepsilon_{0} \approx 8.85 \cdot 10^{-12} \Phi / \mathrm{M}$ - электрическая постоянная.

Глубина потециальной ямы $U_{0}$ для наших целей несущественна, важен сам факт туннелирования, поскольку преодоление барьера предполагает образование электронной пары за счет контактного взаимодействия.

Оценим коэффициент прозрачности для движущегося электрона при туннелировании сквозь барьер, создаваемый одиночным покоящимся электроном с элементарным зарядом $e=1.6 \cdot 10^{-19}$ Кл. Разумеется, предположение о покоящемся электроне носит условный характер. Напомним, типичные скорости электронов в атоме близки к $v \approx 10^{6} \mathrm{~m} / \mathrm{c}$, и, следовательно, для кинетической энергии электронов с массой покоя $m_{0} \approx 9.1 \cdot 10^{-31}$ кг имеем

$$
E_{\text {кин }}=m_{0} v^{2} / 2 \approx 5 \cdot 10^{-19} \text { Дж } \approx 3 \text { эВ. }
$$

Ясно, что условна и фиксация позиции электрона даже с точностью до локализации электрона в объеме атома. Действительно, при зарядовом числе $Z$ локализация электрона в объеме атома возможна лишь при энергии внешнего воздействия, не превышающей

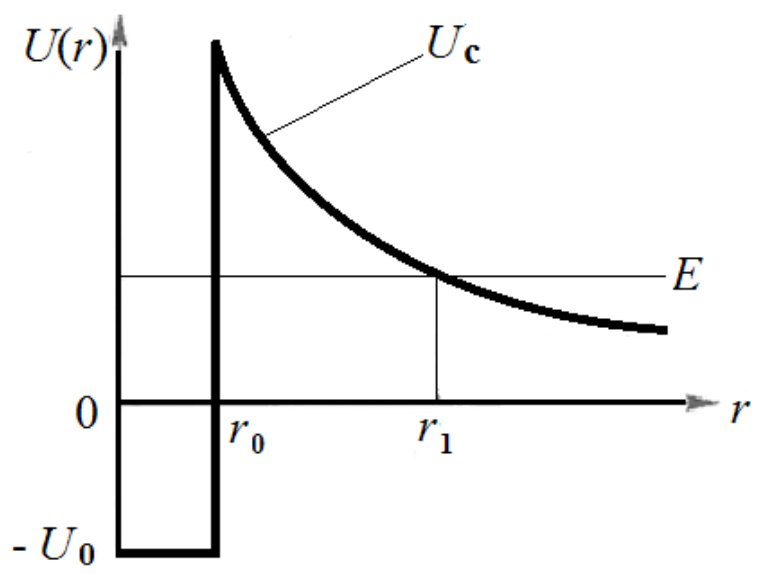

Рис. 2. Модельный потенциал для оценки коэффициента прозрачности при туннелирования электрона с энергией $E$ сквозь кулоновский барьер.

Fig. 2. Model potential for assessing the transparency coefficient during tunneling of an electron with energy $E$ through the Coulomb barrier. энергию ионизации атома $E_{\text {iа }}$, которая примерно в $Z$ раз больше энергии ионизации атома водорода $E_{\mathrm{iH}}$ :

$$
E_{\mathrm{ia}} \approx Z E_{\mathrm{iH}} \approx Z \cdot 13.6 \text { эВ. }
$$

Например, для кислорода $(Z=8) E_{\mathrm{iO}} \approx 109$ эВ. То есть для спаривания с электроном атома кислорода (электрон «мишени») уместно рассматривать внешние электроны с энергими в пределах до 100 эВ. Очевидно, что наиболее выгодным для образования электронных пар является момент локализации электронов «мишени» в области между сталкивающимися атомами (ионами). Электроны мишени могут принадлежать и атомам кислорода, входящим в молекулы кластеризованной воды, для которой характерны тетраэдрические кластеры $[15,16]$ из четырех или даже пяти молекул (в случае объемно центрированного тетраэдра). Таким образом, полагаем электрон мишени находящимся в связанном (атомарном) состоянии. Начало отсчета потенциала выбирается в бесконечно удаленной точке, полуширина прямоугольной ямы $r_{0}$ полагается равной $10^{-15}$ м. Тогда высота энергетического барьера на краю ямы при $r=r_{0}=10^{-15} \mathrm{M}$

$$
U\left(r_{0}\right)=U_{\max }=e^{2} / 4 \pi \varepsilon_{0} r_{0} \approx 1.2 \mathrm{MэB} .
$$

Считаем, что электрон проходит ускоряющую разность потенциалов, приобретая кинетическую энергию $E$ равную $U\left(r_{1}\right)$, при $r_{1}>r_{0}$.

Коэффициент прозрачности $D$, согласно [10], выберем в виде

$$
D=\exp -\frac{2}{\hbar} \int_{r_{0}}^{r_{1}} d r \sqrt{2 m(U(r)-E)}, \quad E=U\left(r_{1}\right) .
$$

С учетом того, что рассматривается взаимодействие пары электронов, естественно использовать систему отсчета, связанную с их центром масс. Тогда величина $m$ соответствует приведенной массе $m_{0} / 2$ и

$$
D=\exp -\frac{e}{\hbar} \sqrt{\frac{m_{0}}{\pi \varepsilon_{0}}} \int_{r_{0}}^{r_{1}} d r \sqrt{\left(\frac{1}{r}-\frac{1}{r_{1}}\right)} .
$$

Интеграл в показателе экспоненты (5) берется точно

$$
\int_{r_{0}}^{r_{1}} d r \sqrt{\left(\frac{1}{r}-\frac{1}{r_{1}}\right)}=-r_{0} \sqrt{\frac{1}{r_{0}}-\frac{1}{r_{1}}}+\sqrt{r_{1}} \operatorname{arctg}\left(\sqrt{\frac{r_{1}}{r_{0}}-1}\right) .
$$

Формально при $r_{0}=r_{1}$ интеграл (6) обращается в ноль, a $D=1$, как и должно быть. Учитывая, что $r_{0} \ll r_{1}$, получаем из (6)

$$
\int_{r_{0}}^{r_{1}} d r \sqrt{\left(\frac{1}{r}-\frac{1}{r_{1}}\right)} \approx \sqrt{r_{1}} \operatorname{arctg}\left(\sqrt{\frac{r_{1}}{r_{0}}}\right) \approx \frac{\pi}{2} \sqrt{r_{1}},
$$

а из (5) и (7)

$$
D \approx\left(\exp -\frac{e}{2 \hbar} \sqrt{\frac{\pi m_{0} r_{1}}{\varepsilon_{0}}}\right)
$$

Допустим, для иллюстрации, что $E=100$ эВ, тогда из (1) находим $r_{1} \approx 1.44 \cdot 10^{-11} \mathrm{M}$, а из $(8) D \approx 0.1932$. 
Таким образом, при $E=100$ эВ прозрачность барьера уже велика $\approx 0.2$.

Руководствуясь значениями $E_{\text {iа }}$ в качестве верхнего предела для существования связанного состояния электрона-мишени, учтем, что в ходе синтеза в [6] возникали массивные ядра вплоть до $\mathrm{Bi}(Z=83)$. При парных реакциях синтеза это возможно, как отмечалось в [8], если массивные элементы образуются из продуктов синтеза предшествующего поколения элементов.

Очевидно, что рост $E_{\text {ia }}$ позволяет рассматривать значения $E>100$ эВ, что сопровождается ростом коэффициента прозрачности. Для удобства читателей результаты расчета приведены в Табл. 1.

Последняя колонка приведена для сравнения, демонстрируя, что снижение $r_{1}$ на порядок (при повышении на порядок E) сопровождается практически трехкратным ростом прозрачности барьера.

\section{3. Обсуждение результатов}

Прежде всего отметим, что приведенные в Табл. 1 оценки значений $D$ велики на фоне значений прозрачности кулоновских барьеров, принимаемых, например, при канонических реакциях синтеза гелия $[1,2]$, при сближении ядер дейтерия и трития до расстояния $R_{c} \sim 10^{-13}$ м. Используя далее тот же вид потенциала, при рассмотрении туннелирования нуклонов вместо малых $r_{0}$ и $r_{1}$ будем использовать $R_{0}$ и $R_{1}$. Действительно, если считать радиус ядерных сил $R_{0}$ порядка фемтометра $R_{n} \sim 10^{-15} \mathrm{M}$, то условие $R_{c} / R_{n} \sim R_{1} / R_{0} \sim r_{1} / r_{0} \gg 1$ выполняется, и можно использовать для оценки $D$ формулу (8), подставляя вместо приведенной массы $m_{0} / 2$ приведенную массу

$$
m \approx\left(2 m_{N}\right)\left(3 m_{N}\right) /\left(2 m_{N}+3 m_{N}\right)=1.2 m_{N}
$$

$\left(m_{N}-\right.$ масса нуклона $\left.m_{N} \approx m_{n} \approx m_{p} \approx 1837 m_{0}\right)$. Согласно [1], полагаем $R_{1}=5 \cdot 10^{-13}$ м (диаметр ионизованной мезомолекулы водорода с ядрами дейтерия и трития, содержащей мюон), тогда из (8), получаем $D=D_{\mu} \approx 1.46 \cdot 10^{-9}$ для процесса туннелирования изотопов водорода. Такие малые значения $D$ контрастируют с данными о быстром протекании (за время $\sim 10^{-12}$ с) реакции синтеза. Скорее всего, это свидетельствует в пользу радиуса межнуклонного взаимодействия за счет обмена квазипозитрониями [8,17] на два порядка превышающего $R_{n}$. Таким образом, естественно полагать, что границе сильного взаимодействия $R_{0}$ соответствует пространственный масштаб порядка $R_{c}$. При $R_{0}=10^{-13} \mathrm{M}$ и том же значении $R_{1}=5 \cdot 10^{-13} \mathrm{M}=5 R_{0}$, используя точное значение интеграла (6), получаем

$$
\int_{R_{0}}^{R_{1}} d r \sqrt{\left(\frac{1}{r}-\frac{1}{R_{1}}\right)} \approx 1.5812326 \sqrt{R_{0}}, D_{\mu} \approx 1.054 \cdot 10^{-4},
$$

то есть $D_{\mu}$ возрастает на пять порядков. Формальное увеличение $R_{0}$ в три раза (до $3 \cdot 10^{-13}$ м) увеличивает $D_{\mu}$ на порядок (до $\left.\approx 1.167 \cdot 10^{-3}\right)$, соответственно при $R_{0}=4 \cdot 10^{-13}$ м значение $D_{\mu} \approx 9.019 \cdot 10^{-3}$ становится близким к 0.01 .

Рассмотрим теперь для сравнения потенциал $H(r)$, представляющий суперпозицию потенциалов Юкавы $U_{\mathrm{y}}$ и Кулона $U_{\mathrm{C}}$. Потенциал притяжения Юкавы имеет вид

$$
U_{\mathrm{Y}}(r)=-g \exp \left(-r / R_{0}\right) / r, \quad g>0 .
$$

Доопределим константу $g$, требуя, чтобы при $r=R_{0}$ выполнялась точная компенсация $U_{\mathrm{Y}}$ и $U_{\mathrm{C}}$, и, следовательно, $H\left(R_{0}\right)=0$. Тогда получаем

$$
H(r)=\frac{e^{2}}{4 \pi \varepsilon_{0} r}\left[1-\exp \left(1-\frac{r}{R_{0}}\right)\right] .
$$

Из (12) очевидно, что при $r>r_{0} H(r)>0$, а при $r<r_{0}$ $H(r)<0$. На Рис. 3 приведена форма потенциала (12) в безразмерных переменных.

На Рис. 3 максимуму функции $H(x)$ соответствует решение уравнения

$$
\exp (1-x)=1 /(1+x)
$$

при $x=x_{\mathrm{m}} \approx 2.1462$ и $H(x)_{\max }=H\left(x_{\mathrm{m}}\right) \approx 0.3178$. Сглаженный (по сравнению с потенциалом на Рис. 2) потенциал $H(x)$ имеет более высокую проницаемость барьера. Так, например, при выборе $R_{0}=10^{-13}$ м и $R_{1}=5 R_{0}$ интеграл от $H(x)$ в пределах от $x_{0} \approx 1.293$ до $x_{1}=5$ равен $\approx 0.917$ вместо $\approx 1.581$ в (10), и в результате значение $D_{\mu}$ возрастает от значения $\approx 1.054 \cdot 10^{-4}$ до $\approx 4.938 \cdot 10^{-3}$, то есть более чем на порядок. Если формально величину $R_{0}$ удвоить, не изменяя $R_{1}=5 \cdot 10^{-13}$ м, то значение $x_{1}=2.5$, и, как очевидно из Рис. 3 , барьер становится практически прозрачным. Однако элементарная оценка [8] свидетельствует в пользу величины $R_{0} \approx 10^{-13}$ м. Следовательно (при $R_{0}=10^{-13} \mathrm{M}$ ), факт быстрого туннелирования ядер водорода указывает на достаточность значения $D_{\mu} \approx 5 \cdot 10^{-3}$ для эффективности реакции синтеза гелия в реакции мюонного катализа.

Ясно, что значительное влияние на коэффициент прозрачности при переходе от потенциала притяжения в форме прямоугольной ямы к потенциалу Юкавы

Табл. 1. Значения $D$ для диапазона энергий $E$ от $10^{2}$ до $10^{3}$ эВ.

Table 1. Values of $D$ for $E$ energies range from $10^{2}$ to $10^{3} \mathrm{eV}$.

\begin{tabular}{|c|c|c|c|c|c|c|c|}
\hline $\begin{array}{c}E, \ni \mathrm{B} \\
E, \mathrm{eV}\end{array}$ & 100 & 144 & 200 & 300 & 400 & 500 & 1000 \\
\hline $\begin{array}{c}r_{1}, 10^{-10} \mathrm{M} \\
r_{1}, 10^{-10} \mathrm{~m}\end{array}$ & 1.44 & 1 & 0.72 & 0.48 & 0.36 & 0.288 & 0.144 \\
\hline$D$ & 0.1932 & 0.2541 & 0.3127 & 0.3870 & 0.4395 & 0.4794 & 0.5946 \\
\hline
\end{tabular}


Табл. 2. Значения $D$ для диапазона энергий $E$ от 10 до $10^{2}$ эВ.

Table 2. Values of $D$ for $E$ energies range from 10 to $10^{2} \mathrm{eV}$.

\begin{tabular}{|c|c|c|c|c|c|c|c|}
\hline $\begin{array}{c}E, \ni \mathrm{B} \\
E, \mathrm{eV}\end{array}$ & 10 & 14.4 & 20 & 30 & 40 & 50 & 100 \\
\hline $\begin{array}{c}r_{1}, 10^{-10} \mathrm{M} \\
r_{1}, 10^{-10} \mathrm{~m}\end{array}$ & 1.44 & 1 & 0.72 & 0.48 & 0.36 & 0.288 & 0.144 \\
\hline$D, 10^{-2}$ & 0.5520 & 1.313 & 2.531 & 4.970 & 7.430 & 9.776 & 19.317 \\
\hline
\end{tabular}

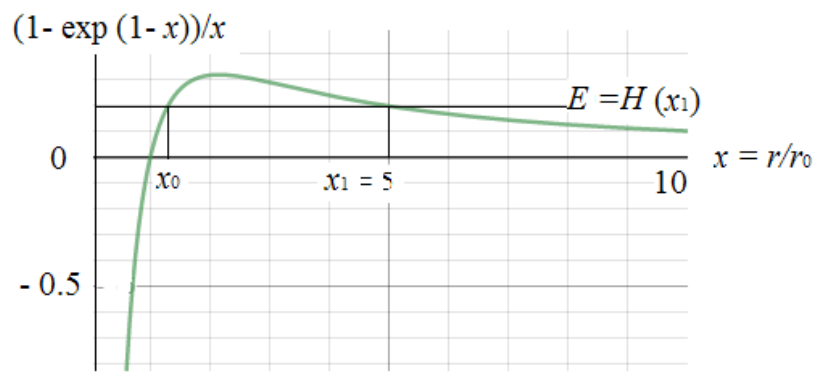

Рис. 3. Потенциал $H(r)$ в безразмерных переменных.

Fig. 3. The potential $H(r)$ in dimensionless variables.

имеет место лишь при значениях $R_{0}$ и $R_{1}$ одного порядка. Поэтому применительно к электронному туннелированию с меньшим на два порядка (по сравнению с $R_{0}=10^{-13} \mathrm{M}$ ) значением $r_{0}=10^{-15}$ м и отличающимся на четыре порядка значением $r_{1}$ переход к модельному суперпозиционному потенциалу $H(r)$ (12) существенных изменений в прозрачности барьера не даст. Важно, что коэффициентам прозрачности $D$ на уровне $10^{-2}-10^{-3}$ для образования электронных пар удовлетворяют энергии $E$ порядка 10 эВ. Но энергии 10 эВ соответствует атомный масштаб $r_{1}=1.44 \cdot 10^{-10}$ м и значение $x_{1}=1.44 \cdot 10^{5}$. Для удобства сравнения с данными Табл. 1 в Табл. 2 приводятся оценки $D$ при сниженных на порядок величинах $E$.

По-видимому, значения $D \sim(0.01-0.1)$ приемлемы для эффективного формирования связанных электронных пар. Поэтому при наборе внешними электронами (в составе тока при электролизе воды) энергии от 10 до 50 эВ следует ожидать появления атомов (и ионов) кислорода с большим содержанием электронных пар, подчиняющихся статистике Бозе-Эйнштейна. Эти пары способны сформировать состояние ПКС с высокой электронной плотностью в межъядерном пространстве и расстоянием $R_{c} \approx R_{0}$, обеспечивающем практически безбарьерное последующее слияние ядер. Заметим, что при таком механизме синтеза не требуется допущения о формировании псевдоядер [18].

Поскольку в конденсированной среде, из-за малых длин свободного пробега электронов, набор энергии $E$ порядка десятков (и сотен) эВ маловероятен, эффективны лишь состояния газовой фазы (типа «плазмоида» в [6]).

Ясно также, что важна и высокая плотность тока, увеличивающая вероятность захвата внешних электронов электронами мишени.

Подчеркнем, ранее в [8] образование контактно связанных пар электронов допускалось как следствие глубоконеупругого столкновения ионов. Проведенный анализ позволяет, на наш взгляд, внести уточнение, акцентировав внимание на роли электронной компоненты электрического тока, способствующей обогащению связанными электронными парами электронных оболочек ионов еще до их столкновения между собой (как и в ходе столкновения). Это принципиально важное в физическом отношении условие существенно для понимания механизма формирования ПКС.

Заметим дополнительно, что значительная плотность тока существенна для формирования квазинейтронных $(p+e)$ состояний [17], включающих в качестве предельных состояний «нейтроиды» Сантилли $[18,19]$ и «гидрино» Миллса [20,21]. Существование квазинейтронов в рамках простой методики электролиза [7,12-14], сопровождающейся экстракцией материала электродов с ядрами $\mathrm{X}^{A}$, должно приводить к элементарным реакциям

$$
{ }_{Z} \mathrm{X}^{A}+(p+e) \rightarrow{ }_{Z+1} \mathrm{Y}^{A+1}+e
$$

при захвате ядром $\mathrm{X}^{A}$ протона $p$, «эскортированного» электроном, либо

$$
\mathrm{X}^{A}+(p+e) \rightarrow{ }_{Z-1} \mathrm{Y}^{A}+p+v
$$

при захвате ядром ${ }_{Z} \mathrm{X}^{A}$ электрона из квазинейтрона ( $v-$ электронное нейтрино). Возможен, конечно, и вариант формирования изотопа:

$$
{ }_{Z} \mathrm{X}^{A}+(p+e) \rightarrow{ }_{Z} \mathrm{X}^{A+1} .
$$

Например, при медных электродах следует ожидать синтеза цинка. Подобные закономерности свидетельствовали бы в пользу возникновения квазинейтронных состояний в процессе плазменного электролиза воды.

\section{4. Заключение}

1. Оценка коэффициента прозрачности $D$ в случае туннелирования электронов при образовании контактно связанных на фемтомасшабе электронных пар показывает, что достаточно высокие значения $D \sim(0.01-0.1)$ достигаются уже для энергий $(10-50)$ эВ внешних электронов.

2. В случае плазменного электролиза воды и водных растворов при разностях потенциалов между электродами порядка сотен вольт, набор электронами энергий в десятки эВ осуществляется в газообразной фазе.

3. Электронная компонента тока при электролизе способствует предварительному (до столкновения) обогащению электронных оболочек ионов, участвую- 
щих в синтезе химических элементов, связанными электронными парами, что существенно для образования ПКС, обеспечивающего сближение ядер до расстояний $\sim 10^{-13}$ м, соответствующих верхней границе сильного взаимодействия.

Благодарности/Acknowledgements. Авторь признательны члену-корреспонденту РАН В.Ф. Балакиреву за обсуждение результатов работы. /The authors are grateful to V.F. Balakirev, Corresponding Member of the Russian Academy of Sciences for discussing the results of the work.

\section{Литература/References}

1. S. S. Gershtein, Yu. V. Petrov, L. I. Ponomarev. Sov. Phys. Usp. 33 (8), 591 (1990). Crossref

2. L. I. Men'shikov, L. N. Somov. Sov. Phys. Usp. 33 (8), 616 (1990). Crossref

3. M.P. Kashchenko, V.F. Balakirev. Letters on materials. 7 (4), 380 (2017). Crossref

4. V.V. Krymskiy, V.F. Balakirev. Doklady Physical Chemistry. 385 (4-6), 197 (2002). Crossref

5. V. V. Krymskiy, V.F. Balakirev, N. V. Plotnikova. J. Chem. Chem. Eng. 9 (3), 211 (2015). Crossref

6. V.F. Balakirev, V.V. Krymskiy, B.V. Bolotov et al. Interconversion of chemical elements. Ekaterinburg, UB RAS (2003) 97 p. (in Russian) [В.Ф. Балакирев, В. Крымский, Б. В. Болотов и др. Взаимопревращения химических элементов. Екатеринбург, УрО РАН (2003) 97 c.]

7. V.A. Pan'kov, B. P. Kuzmin. Actual problems of modern science. 5, 117 (2008). (in Russian) [В.А. Паньков, Б.П. Кузьмин. Актуальные проблемы современной науки. 5, 117 (2008).]

8. M.P. Kashchenko, V.F. Balakirev. Letters on materials. 8 (2), 152 (2018). (in Russian) [М.П. Кащенко, В.Ф. Балакирев. Письма о материалах. 8 (2), 152 (2018).] Crossref

9. R.M. Santilli. Foundations of Hadronic Chemistry. With Applications to New Clean Energies and Fuels. London, Kluwer Academic Publishers (2001) 554 p.

10. L. D. Landau, E. M. Lifshitz. Quantum Mechanics: NonRelativistic Theory, 3rd ed. Pergamon Press Ltd., Oxford (1977) 667p.

11. H. Yukawa. Proc. Phys. Math. Soc. Japan. 17 (2), 48 (1935).
12. M.P. Kashchenko, V.F. Balakirev, M.B. Smirnov, Yu.L. Chepelev, V.V. Ilushin, N.V. Nikolaeva, V.G. Pushin. International conference "Materials science of the future: research, development, scientific training". Nizhni Novgorod (2019) p. 40.

13. M.P. Kashchenko, V.F. Balakirev, M.B. Smirnov, Yu. L. Chepelev, V.V. Ilushin, N.V. Nikolaeva, V.G. Pushin. The thirteenth international Ural seminar "Radiation damage physics of metals and alloys". Kyshtym, Russia (2019) p. 68. (in Russian) [М.П. Кащенко, В.Ф. Балакирев, М.Б. Смирнов, Ю.Л. Чепелев, В.В. Илюшин, Н.В. Николаева, В.Г. Пушин. Тринадцатый международный уральский семинар «Радиационная физика металлов и сплавов». Кыштым, Россия (2019) с. 68.]

14. M.P. Kashchenko, V.F. Balakirev, N.M. Kashchenko, M.B. Smirnov, Yu.L. Chepelev, V.V. Ilushin, N. V. Nikolaeva, V. G. Pushin. Letters on materials. 10 (1), 66 (2020). (in Russian) [М. П. Кащенко, В. Ф. Балакирев, Н.М. Кащенко, М.Б. Смирнов, Ю.Л. Чепелев, В. В. Илюшин, Н. В. Николаева, В. Г. Пушин. Письма о материалах. 10 (1), 66 (2020).] Crossref

15. T. Tokushima, Y. Harada, O. Takahashi, Y. Senba, H. Ohashi, L. G. M. Pettersson, A. Nilsson, S. Shin. Chem. Phys. Lett. 460, 387 (2008). Crossref

16. C. Huang, K. T. Wikfeldt, T. Tokushima, D. Nordlund, Y. Harada, U. Bergmann, M. Niebuhr, T.M. Weiss, Y. Horikawa, M. Leetmaa, M. P. Ljungberg, O. Takahashi, A. Lenz, L. Ojamae, A.P. Lyubartsev, S. Shin, L. G. M. Pettersson, A. Nilsson. PNAS. 106, 15214 (2009). Crossref

17. M.P. Kashchenko, N.M. Kashchenko. Letters on materials. 9 (3), 316 (2019). (in Russian) [М. П. Кащенко, Н.М. Кащенко. Письма о материалах. 9 (3), 316 (2019).] Crossref

18. R.M. Santilli. International Journal of Applied Physics and Mathematics. 9 (2), 72 (2019). Crossref

19. R. Norman, A. A. Bhalekar, S. Beghella, B. B. Buckley, J. Dunning-Davies, J. Rak, R. M. Santilli. American Journal of Modern Physics. 6 (4-1), 85 (2017). Crossref

20. R.L. Mills. The Grand Unified Theory of Classical Physics. BlackLight Power, Inc., Cranbury, New Jersey (2011).

21. R. Mills, Y. Lu, R. Frazer. Chinese Journal of Physics. 56, 1667 (2018). $\underline{\text { Crossref }}$ 